\title{
Sclerosing angiomatoid nodular transformation of the spleen: A case report and literature review
}

\author{
TIAN-BAO WANG ${ }^{1 *}$, BAO-GUANG HU ${ }^{2 *}$, DA-WEI LIU ${ }^{3}$, ZHEN-HUA GAO ${ }^{4}$, \\ HAN-PING SHI ${ }^{1}$ and WEN-GUANG DONG ${ }^{1}$ \\ ${ }^{1}$ Department of Surgery, The First Affiliated Hospital, Sun Yat-sen University, Guangzhou, Guangdong 510080; \\ ${ }^{2}$ Department of Gastrointestinal Surgery, The Affiliated Hospital, Binzhou Medical College, Binzhou, \\ Shandong 256603; Departments of ${ }^{3}$ Pathology and ${ }^{4}$ Radiology, The First Affiliated Hospital, \\ Sun Yat-sen University, Guangzhou, Guangdong 510080, P.R. China
}

Received April 2, 2015; Accepted May 17, 2016

DOI: $10.3892 / \mathrm{ol} .2016 .4720$

\begin{abstract}
Sclerosing angiomatoid nodular transformation (SANT) is a rare benign splenic vascular lesion. Since it was first defined in 2004, a total of 132 cases of SANT have been reported in 50 studies in the English literature. However, it remains difficult to form a definitive pre-operative differential diagnosis of SANT compared with other splenic tumors or malignant lesions. The present study reports a pathologically proven case of SANT in a 29-year-old man who initially presented with left upper quadrant and back discomfort. The study also provides a review of the current knowledge on the condition, including the clinical profile, imaging features, cytological features, differential diagnosis and treatment of SANT. The most important distinguishing features of SANT are its typical vascular character and lack of other features that are typical of a granuloma. A splenectomy is required and the diagnosis is based on pathological analysis.
\end{abstract}

\section{Introduction}

Sclerosing angiomatoid nodular transformation of the spleen (SANT) is an extremely rare benign lesion of the spleen $(1,2)$, which was first characterized by Martel et al (1) in 2004. To date, a total of 133 cases of SANT have been reported in the English literature; however, the exact incidence rate remains unclear. The majority of patients are asymptomatic at presentation and SANT is identified incidentally on imaging. For

Correspondence to: Professor Tian-Bao Wang, Department of Surgery, The First Affiliated Hospital, Sun Yat-sen University, 58 Zhongshan Second Road, Guangzhou, Guangdong 510080, P.R. China

E-mail: zsdxwtb@163.com

*Contributed equally

Key words: sclerosing angiomatoid nodular transformation, spleen, diagnosis, treatment symptomatic patients, common presentations are incidental splenic mass, abdominal discomfort or pain. There is a slight female preponderance and middle-aged adults are predominantly affected (1). The majority of cases undergo traditional or laparoscopic splenectomy, and diagnosis is confirmed using histopathological examination. No recurrence of SANT has been reported following a splenectomy; therefore, traditional or laparoscopic splenectomy is considered to be a curative technique for the management of SANT (2). By contrast, due to absent definitive imaging signs and varying growth patterns, it remains challenging to form a clearly pre-operative diagnosis of SANT compared with other splenic tumors or malignant lesions. The present study reports a novel case of SANT and discusses the characteristics of imaging, immunohistochemical profile and differential diagnosis, which may result in improved management of SANT.

\section{Case report}

A 29-year-old man presented to the Department of Surgery, The First Affiliated Hospital, Sun Yat-sen University (Guangzhou, China) on March 30, 2014 with a 1-year history of left upper quadrant and back discomfort. The discomfort was intermittent, but had become progressively worse since it first occurred. There was no history of fever, weight loss and other medical issues, and the patient's respiratory, digestive and urinary systems were normal. Abdominal examination found no positive physical signs. Hematological parameters, and liver and kidney function tests were unremarkable. On computed tomography (CT) scan, a round mass of 76x74 mm was observed in the spleen, which showed slightly low density on a plain scanning CT image (Fig. 1A). CT values of the mass and the surrounding normal splenic tissue were $\sim 40$ and $42 \mathrm{HU}$, respectively, prior to the injection of contrast medium. The mass showed heterogeneous moderate enhancement with a value of $66 \mathrm{HU}$, which was clearly lower than that of the surrounding normal spleen (136 HU) on arterial phase-enhanced CT scanning. Certain regions within the lesion were significantly enhanced (Fig. 1B). The CT value of the mass was slightly increased (80 HU) on portal venous phase-enhanced CT scanning, but remained lower than that of the surrounding spleen (116 HU) (Fig. 1C). 
A

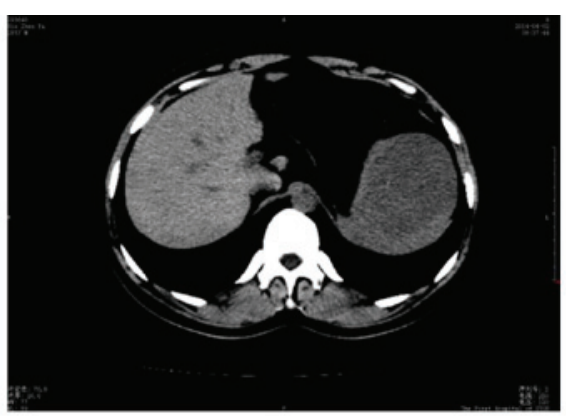

C

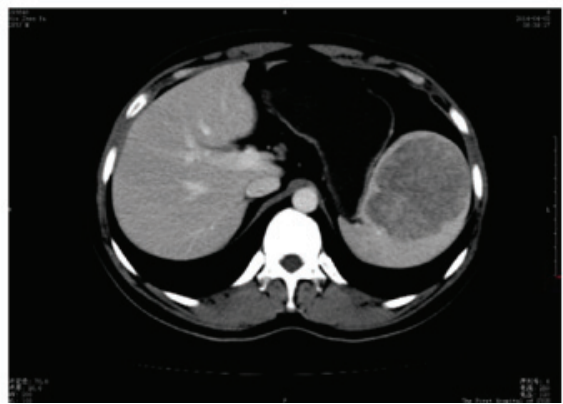

B
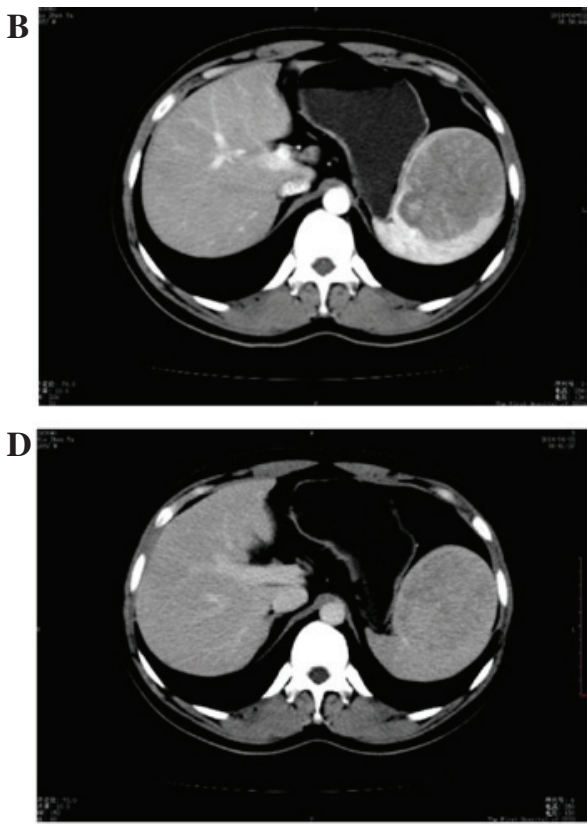

Figure 1. CT scans of sclerosing angiomatoid nodular transformation. (A) Slightly low density lesion on a plain scanning CT image. (B) The CT value of the mass was clearly lower than that of the surrounding normal spleen on arterial phase-enhanced CT scanning. (C) The CT value of the mass remained lower than that of the surrounding spleen on portal venous-phase enhanced CT scanning. (D) The densities of the mass and the normal surrounding spleen were close on delayed enhanced CT scanning (D). CT, computed tomography.

The densities of the mass and the normal surrounding spleen were close on delayed enhanced CT scanning after a delay of $3 \mathrm{~min}$ (Fig. 1D). The patient underwent a splenectomy. A well-circumscribed mass measuring $7 \mathrm{~cm}$ in diameter was noted in the cross-sections of the resected specimen. On gross examination, the mass exhibited a gray-white or gray-red cut surface with fibrous septa traversing throughout and was divided into discrete complete or incomplete nodules.

Resected tissues underwent routine preparation for hematoxylin and eosin staining, and light microscopy confirmed the nodular appearance observed during the gross examination. Observation under a light-contrast microscope showed that the nodules were separated by fibrous or fibrosclerotic stroma with hemosiderophages, fibroblasts and lympho-mononuclear cells (Fig. 2A). Within the nodules, extravasated erythrocytes and hemosiderin pigment were plentiful, and oval stromal cells, red blood cells and mild chronic inflammatory cell infiltration were also present (Fig. 2B). In addition, three distinct types of blood vessels were also observed: Splenic cord-type capillaries, sinusoid-type spaces and small veins. The results of immunohistochemistry tests revealed that the endothelial cells in these blood vessels showed heterogenous immunohistological profiles of cluster of differentiation (CD)8-, CD31- and CD34-positive cells, which indicated their derivation from sinusoidal, capillary-like and vein-like elements (Fig. 2C-E). Immunohistochemistry was performed using the following rabbit polyclonal antibodies, which were purchased from Abcam (Cambridge, MA, USA): Anti-CD8 (catalog no., ab4055; dilution, 1:200), anti-CD31 (catalog no., ab28364; dilution, 1:50) and anti-CD34 (catalog no., ab110643; dilution, 1:200) Horseradish peroxidase and intelliPATH FLXDAB DAB Chromogen kit (Biocare Medical, Inc., Concord, CA, USA) were used for visualization. These findings and the characteristic macroscopic features led to the diagnosis of SANT.
The patient remained asymptomatic and there was no evidence of recurrence subsequent to a follow-up period of 13 months. Written informed consent was obtained from the patient for the publication of the present study.

\section{Discussion}

The prevalence of splenic tumors is rare, and the majority are vascular or inflammatory in origin $(3,4)$. As a novel entity that differed from other splenic vascular tumors, SANT was first defined by Martel et al (1) in 2004. However, prior to its identification, this lesion was possibly reported in other studies under the names of different splenic vascular lesions, such as hamartoma, hemangioma or hemangioendothelioma (4). The present study reviews the current knowledge on SANT, beginning with the present case, and discusses other cases reported in the medical English literature.

In a review of the existing English literature, a total of 133 cases of SANT have been found to be reported in $\sim 50$ studies after the condition was first defined in 2004 (2). The cases consisted of 59 males and 74 females, with a female-to-male ratio of $1.25: 1$, which is similar to the results recently reported by Falk et al in 2012 (2), but lower than the ratio of 2:1 reported by Diebold et al in 16 splenectomy specimens (5). Therefore, the gender ratio may change as more cases are reported. The median age of the patients in the present literature review was 44 years, and the majority (94/133 cases) were aged between 30 and 60 years. Thus, SANT predominantly affects the middle-aged population. In these cases, $>80$ lesions were asymptomatic and were found incidentally during imaging. For symptomatic patients, abdominal pain or discomfort was predominant, but certain presentations included a palpable mass, flank pain, pelvic pain, anemia, thrombocytopenia, long-standing fevers and night sweats. 

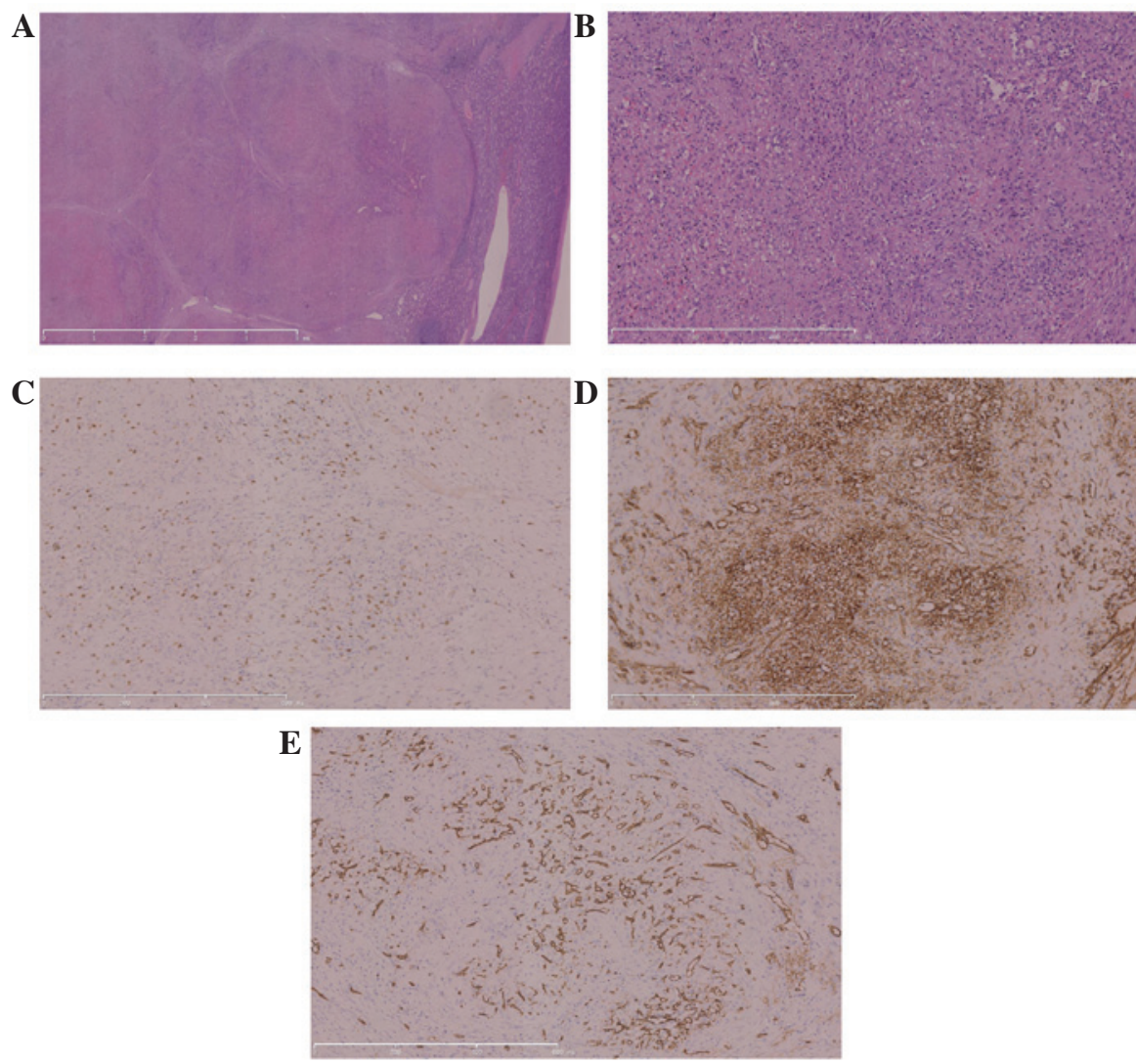

Figure 2. Pathological examination of sclerosing angiomatoid nodular transformation. (A) Nodules were separated by fibrous or fibrosclerotic stroma (magnification, $x 12.5)$. (B) Within the nodules, extravasated erythrocytes and hemosiderin pigment were plentiful. Immunohistochemical analysis documented positive expression of (C) CD8, (D) CD31 and (E) CD34 (magnification, x100). CD, cluster of differentiation.

Pathological features were the key for the diagnosis of SANT, as originally described by Martel et al (1) in a series of 25 patients and confirmed in multiple subsequent case series by other researchers. The present literature review summarizes these features as follows: Firstly, the size of the lesion varied widely from $0.1-17 \mathrm{~cm}$ in diameter among the 133 cases of SANT. Secondly, gross examination showed a well-circumscribed but non-encapsulated bosselated mass, with multiple individual or confluent red-brown or tan-white nodules separated by whitish fibrotic stroma. In addition, the lesion was slightly firmer than the rest of the spleen. This classic appearance is diagnostic for SANT (6). Moreover, as shown in Fig. 1, the microscopic examination of SANT usually reveals a multinodular growth pattern composed of a variable mixture and sieve-like arrangement of vascular spaces that are often surrounded by dense collagen fibrosis or fibroid rims. The circumscription of nodules by collagen and the nature of the intervening stroma can vary among individual tumors. Centrally, the nodules contain vascular channels of varying sizes lined with thick endothelium interspersed with ovoid or spindle cells. Numerous erythrocytes, myofibroblasts, plasma cells, siderophages, and inflammatory cells can appear in these nodules. Notably, no markedly cytological atypia, mitotic figures, histiocytic giant cells or necrosis were observed in the lesions.

The classic appearance of SANT with regard to the immunohistochemical profiles includes three distinct types of blood vessels and endothelial cells stained with CD34, CD8 or CD31, respectively (7). The first type of vessels consists of well-formed cord capillaries in an organized lobular arrangement in endothelial cells positive for CD34 and CD31, but negative for CD8. The second type of vessels is consistent with splenic sinusoids and includes cells negative for CD34, but positive for CD31 and CD8. The third type consists of small veins that are arranged in intricate mesh-like patterns and include cells negative for CD34 and CD8, but positive for CD31. In the studies reviewed, these classic appearances were observed in almost all specimens (7). Moreover, none of the cells in the lesions stained positively for CD23, anaplastic lymphoma kinase, CD30, CD68 or Epstein-Barr virus latent membrane protein 1 .

CT imaging cannot specifically distinguish SANT from various other types of vascular lesions in the spleen, but it is the major method to find SANT lesions (8). In the 133 cases of SANT identified in the present review, CT imaging was performed in almost all of the patients, and the CT features of SANT were relatively unique. As described in a previous study (9), SANT generally presents as a well-circumscribed, solitary, isodense or hypodense mass in the spleen on plain CT scanning images in the portal and late portal venous phase, with or without a small central calcification. In multiphase imaging, a number of SANT lesions showed a 'spoke-and-wheel' pattern (10). Detailed study of radiological and pathological findings revealed that this spoke-and-wheel morphology corresponded to a central, stellate fibrous stroma with fibrous septa that separated angiomatoid nodules (9). Moreover, positron-emission tomography-CT and 
single-photon emission CT/CT (SPECT/CT) were also used for the diagnosis of SANT. As a benign lesion, SANT should not be avid for ${ }^{18} \mathrm{~F}$-fluorodeoxyglucose (FDG) (11). However, at least 2 cases of SANT showed mild to moderate FDG avidity, and the lesion appeared as a hypermetabolic-activity mass in the FDG-positron emission tomography image, simulating a neoplasm $(11,12)$. This feature suggests that SANT lesions may have a higher glycolytic activity than the surrounding splenic parenchyma. Moreover, only 1 study (11) described a finding of SANT based on ${ }^{99 \mathrm{~m}} \mathrm{Tc}$-sulfur colloid SPECT/CT imaging, and normal tracer activity was observed within the liver and spleen, but not within the splenic lesion. This appearance confirmed the absence of reticuloendothelial elements within splenic SANT.

Only a small number of cases of SANT included magnetic resonance imaging (MRI) findings, which limits any broad statements regarding the MRI imaging of SANT. Karaosmanoglu et al (10) first described the MRI findings of SANT, and reported that the lesion presented as a central hyperintense area that was consistent with hemorrhage on fat-saturated precontrast T1-weighted images. However, on T2-weighted images, the lesion appeared as a spoke-and-wheel pattern, which was similar to the pattern obtained by multiphase imaging with CT. Subsequent studies $(1,12-14)$ have described the MRI features of SANT, and numerous investigators have suggested that the spoke-and-wheel pattern from MRI and multiphase CT imaging may be useful for the diagnosis of SANT.

Only a few studies on ultrasonography for the diagnosis of SANT have appeared in the English literature, and therefore, the sonographic features of SANT are poorly described (15-19). On gray-scale ultrasonography, SANT usually appears as a heterogeneously hypoechoic mass with bright linear echoes accompanied by acoustic shadowing in the center $(16,17)$. These linear echoes usually show blood signals on color Doppler images and an arterial waveform on spectral Doppler analysis. Contrast-enhanced ultrasound examination has better temporal resolution than contrast-enhanced CT and can dynamically visualize blood-flow distribution in the vascular phase of the tumor. With the aid of ultrasound contrast agents such as Sonazoid (Daiichi-Sankyo, Tokyo, Japan) or SonoVue (Bracco, Milan, Italy), SANT appears as a spoke-and-wheel enhancement that extends radially from the center of the mass during the early vascular phase (16).

CT, MRI and ultrasonography can provide various types of information on SANT, but the images alone are not usually sufficient to radiologically distinguish SANT from other benign splenic lesions. To address this shortcoming, Gutzeit et al (16) first reported the use of ultrasound-guided core needle biopsy (CNB) for the diagnosis of SANT. Although the study suggested that ultrasound-guided CNB is safe, another study (1) reported opposing views due to the danger of bleeding and tumor dissemination. Further imaging studies must be performed to provide more reliable information for the diagnosis of SANT.

The differential diagnosis of SANT involves consideration of several other benign splenic lesions and malignant vascular lesions. These include hemangioma, lymphangioma, littoral cell angioma, hamartoma, angiosarcoma and inflammatory pseudotumor (IPT).
Hemangiomas of the spleen are the most common benign tumors that arise from sinusoidal epithelial cells (20). Usually smaller than $2 \mathrm{~cm}$, the tumors show cavernous configurations on imaging (20). Histologically, the endothelial cells are positive for CD31 and CD34, and are negative for CD8, CD21 and CD68 (4). A characteristic difference is that hemangiomas lack the typical trivascular pattern of proliferation observed in SANT (4).

Lymphangioma is a benign splenic tumor that arises from the lymphatic endothelium (21). The tumor usually presents as either a solitary nodule or a diffuse process, and capillary and cavernous forms also exist (6). Microscopic examination reveals cystic spaces lined with single- or multi-layered endothelium, with occasional papillary excrescences $(2,5,21)$. Immunostaining shows the lymphatic endothelia are strongly positive for D2-40 immunostain (Ventana, Tucson, AZ), and negative for CD21 and CD8 $(2,21)$.

Littoral cell angioma is a vascular tumor that is derived from the littoral cells (22). Histological examination shows that these cells line the splenic sinuses and exhibit both endothelial and histocytic phenotypes (2). Significantly, littoral cell angioma has a characteristic CD34/CD $68^{+} / \mathrm{CD} 21^{+} / \mathrm{CD} 8$ immunophenotype, which is extensively different from that of SANT (2).

Splenic hamartoma is another rare vasoformative lesion of the spleen (23). Microscopically, it displays predominantly red pulpy elements in a disorganized fashion, with scanty fibrous trabeculae (6). On immunohistochemistry, it is positive for factor VIII, CD31, CD8 and type IV collagen, but is negative for CD34, CD21 and CD68 (6). The typical difference between the tumor and SANT is the absence of a nodular growth pattern in splenic hamartoma and the presence of the classical immunophenotype in SANT (2).

Angiosarcoma is the most common malignant primary tumor of the spleen (24). The histological features of angiosarcoma include irregular, anastomosing vascular channels, with marked cytological atypia, brisk mitoses and invasion. The atypical cells have a vascular endothelial phenotype and are positive for CD68 and CD8 (24). The characteristics that differentiate SANT from angiosarcoma include the absence of a nodular growth pattern, and the presence of invasion, cytological atypia and mitosis (2).

IPT is a splenic lesion that is particularly similar to SANT (25). Microscopic examination reveals a mass comprising spindle cells, with features of fibroblasts and myofibroblasts in association with a mixed inflammatory infiltrate and a variable hypocellular fibrocollagenous stroma $(3,25)$. These features are also present in SANT, but IPT lacks angiomatoid nodules and shows a CD34\%/CD8 $/ \mathrm{CD} 21^{-}$phenotype (3).

There is currently no sensitive and specific way to diagnose SANT without a tissue sample, and on the basis of conventional imaging studies, it is pre-operatively difficult to rule out the possibility of a malignant neoplasm. As aforementioned, an ultrasound-guided CNB of the spleen has been used for tissue diagnosis (16), but the problem of bleeding or tumor dissemination may be an unwanted effect of this procedure. Thus, in the majority of cases, splenectomy has become the recommended technique. With the development of laparoscopic instruments and techniques during the past decades, laparoscopic 
surgery has been deployed extensively in numerous surgical fields. Since the introduction of laparoscopic splenectomy by Delaitre and Maignien in 1991 (26), this procedure has been recognized as a safe and effective treatment for certain splenic diseases. To date, $>30$ patients with SANT have undergone laparoscopic splenectomy, and no serious problems have been observed during or after this procedure (27-30). Hence, laparoscopic splenectomy may be a safe and effective procedure for the diagnosis and treatment of SANT, particularly when malignancy cannot be ruled out.

Overall, the present study reports a novel case of SANT that underwent splenectomy successfully. By comparison to other cases, the lesion exhibited no outstanding difference on presentation and characters during imaging. Therefore, when solitary lesions are identified in the spleen during imaging examination, clinicians and radiologists should be conscious of the possibility of SANT. To overcome these challenges, a thorough histopathological examination and immunohistochemical analysis are necessary.

\section{References}

1. Martel M, Cheuk W, Lombardi L, Lifschitz-Mercer B, Chan JK and Rosai J: Sclerosing angiomatoid nodular transformation (SANT): Report of 25 cases of a distinctive benign splenic lesion. Am J Surg Pathol 28: 1268-1279, 2004.

2. Falk GA, Nooli NP, Morris-Stiff G, Plesec TP and Rosenblatt S: Sclerosing angiomatoid nodular transformation (SANT) of the spleen: Case report and review of the literature. Int J Surg Case Rep 3: 492-500, 2012

3. Thipphavong S, Duigenan S, Schindera ST, Gee MS and Philips S: Nonneoplastic, benign, and malignant splenic diseases: Cross-sectional imaging findings and rare disease entities. AJR Am J Roentgenol 203: 315-322, 2014.

4. Önder S, Kosemehmetoglu K, Himmetoglu C, Firat P and Uner A: Sclerosing angiomatoid nodular transformation (SANT) of spleen A case report describing cytology, histology, immunoprofile and differential diagnosis. Cytopathology 23: 129-132, 2012.

5. Diebold J, Le Tourneau A, Marmey B, Prevot S, Müller-Hermelink HK, Sevestre H, Molina T, Billotet C, Gaulard P, Knopf JF, et al: Is sclerosing angiomatoid nodular transformation (SANT) of the splenic red pulp identical to inflammatory pseudotumour? Report of 16 cases. Histopathology 53: 299-310, 2008.

6. Agrawal M, Uppin SG, Bh S, Suppin M, N B and Challa S: Sclerosing angiomatoid nodular transformation of the spleen: A new entity or a new name? Turk Patoloji Derg Apr 92014 (Epub ahead of print)

7. Pradhan D and Mohanty SK: Sclerosing angiomatoid nodular transformation of the spleen. Arch Pathol Lab Med 137: 1309-1312, 2013

8. Raman SP, Singhi A, Horton KM, Hruban RH and Fishman EK Sclerosing angiomatoid nodular transformation of the spleen (SANT): Multimodality imaging appearance of five cases with radiology-pathology correlation. Abdom Imaging 38: 827-834, 2013

9. Lewis RB, Lattin GE Jr, Nandedkar M and Aguilera NS Sclerosing angiomatoid nodular transformation of the spleen: CT and MRI features with pathologic correlation. AJR Am J Roentgenol 200: W353-W360, 2013.

10. Karaosmanoglu DA, Karcaaltincaba M and Akata D: CT and MRI findings of sclerosing angiomatoid nodular transformation of the spleen: Spoke wheel pattern. Korean J Radiol 9 (Suppl): S52-S55, 2008.
11. Thacker C, Korn R, Millstine J, Harvin H, Van Lier Ribbink JA and Gotway MB: Sclerosing angiomatoid nodular transformation of the spleen: CT, MR, PET, and ${ }^{99}(\mathrm{~m}) \mathrm{Tc}$-sulfur colloid SPECT CT findings with gross and histopathological correlation. Abdom Imaging 35: 683-689, 2010.

12. Lee D, Wood B, Formby M and Cho T: F-18 FDG-avid sclerosing angiomatoid nodular transformation (SANT) of the spleen: Case study and literature review. Pathology 39: 181-183, 2007.

13. Bamboat ZM and Masiakos PT: Sclerosing angiomatoid nodular transformation of the spleen in an adolescent with chronic abdominal pain. J Pediatr Surg 45: E13-E16, 2010.

14. Chikkappa MG, Morrison C, Lowe A, Antrim R, Swirsky DM and Gokhale J: Case report and magnetic resonance images of sclerosing angiomatoid nodular transformation (SANT) of the spleen. BMJ Case Rep 2009: bcr07.2009.2131, 2009.

15. Cao JY, Zhang H and Wang WP: Ultrasonography of sclerosing angiomatoid nodular transformation in the spleen. World J Gastroenterol 16: 3727-3730, 2010.

16. Gutzeit A, Stuckmann G and Dommann-Scherrer C: Sclerosing angiomatoid nodular transformation (SANT) of the spleen: Sonographic finding. J Clin Ultrasound 37: 308-311, 2009.

17. Kim HJ, Kim KW, Yu ES, Byun JH, Lee SS, Kim JH and Lee JS: Sclerosing angiomatoid nodular transformation of the spleen: Clinical and radiologic characteristics. Acta Radiol 53: 701-706, 2012.

18. Lee M, Caserta M and Tchelepi H: Sclerosing angiomatoid nodular transformation of the spleen. Ultrasound Q 30: 241-243, 2014.

19. Watanabe M, Shiozawa K, Ikehara T, Kanayama M, Kikuchi Y, Ishii K, Okubo Y, Shibuya K and Sumino Y: A case of sclerosing angiomatoid nodular transformation of the spleen: Correlations between contrast-enhanced ultrasonography and histopathologic findings. J Clin Ultrasound 42: 103-107, 2014.

20. Giovagnoni A, Giorgi C and Goteri G: Tumours of the spleen. Cancer Imaging 5: 73-77, 2005.

21. Singh P, Mathur SK, Singh S, Marwah N, Kalra R and Arora B: Cystic lymphangioma of the spleen-a case report. Indian J Pathol Microbiol 48: 202-203, 2005.

22. Emir S, Sozen S, Yazar MF, Altinsoy HB, Arslan Solmaz O and Ozkan Z: Littoral-cell angioma of the spleen. Arch Iran Med 16: 189-191, 2013.

23. Di Blasi A, Boscaino A, De Dominicis G and Marino-Marsilia G: Splenic hamartoma. Pathologica 97: 124-129, 2005 (In Italian).

24. Delacruz V, Jorda M, Gomez-Fernandez C, Benedetto P and Ganjei P: Fine-needle aspiration diagnosis of angiosarcoma of the spleen: A case report and review of the literature. Arch Pathol Lab Med 129: 1054-1056, 2005.

25. Kim HH, Hur YH, Koh YS, Kim JC, Kim HJ, Kim JW, Kim Y, Lee JH and Cho CK: Sclerosing angiomatoid nodular transformation of the spleen related to IgG4-associated disease: Report of a case. Surg Today 43: 930-936, 2013.

26. Delaitre B and Maignien B: Splenectomy by the laparoscopic approach. Report of a case. Presse Med 20: 2263, 1991 (In French).

27. Budzyński A, Demczuk S, Kumiega B, Migaczewski M, Matłok M and Zub-Pokrowiecka A: Sclerosing angiomatoid nodular transformation of the spleen treated by laparoscopic partial splenectomy. Wideochir Iinne Tech Maloinwazyjne 6: 249-255, 2011

28. Kakisaka T, Kamiyama T, Yokoo H, Orimo T, Wakayama K, Tsuruga Y, Kamachi H, Harada T, Kato F, Yamada Y, et al: Hand-assisted laparoscopic splenectomy for sclerosing angiomatoid nodular transformation of the spleen complicated by chronic disseminated intravascular coagulation: A case report. Asian J Endosc Surg 7: 275-278, 2014.

29. Kim KH, Lee S, Youn SH, Lee MR, Kim MC, Rha SH and Jung GJ: Laparoscopic splenectomy for sclerosing angiomatoid nodular transformation of the spleen. Journal of the Korean Surgical Society 80 (Suppl 1): S59-S62, 2011.

30. Mohr Z, Klippel S, Spiethoff A, Trick D and Willis S: Laparoscopic splenectomy for sclerosing angiomatoid nodular transformation. Chirurg 82: 714-718, 2011 (In German). 